\title{
Water dynamics at the nanoscale
}

\author{
C.Masciovecchio, F.Bencivenga, A.Gessini \\ Sincrotrone Trieste, S.S. 14 km 163,5 in AREA Science Park 34012 Basovizza, Trieste, Italy
}

Received November 12, 2007

The recent construction of an Inelastic UltraViolet Scattering (IUVS) beamline at the ELETTRA Synchrotron Light Laboratory opens new possibilities for studying the density fluctuation spectrum, $S(Q, E)$, of disordered systems in the mesoscopic momentum $(Q)$ and energy $(E)$ transfer region not accessible by other spectroscopic techniques. As an example of possible application of IUVS technique we will discuss the new insights provided in the case of water dynamics. From the analysis of IUVS spectra we were able to measure the temperature and pressure dependencies of structural relaxation time $(\tau)$ in water. In the case of room-pressure water the values of $\tau$, as derived by IUVS, are fairly consistent with previous determinations and, most important, its temperature dependence agrees with Mode Coupling Theory (MCT) predictions. Moreover we found that $\tau$ decreases with increasing pressure at fixed temperature. The observed trend demonstrates that the structural relaxation phenomenology is strongly affected by the applied pressure. However, further investigations are necessary in order to clarify the physical meaning of these preliminary experimental results.

Key words: water, inelastic scattering, relaxation

PACS: $78.35 .+c, 62.60 .+v, 62.50 .+p$

\section{Introduction}

The physics of systems without translational invariance, such as liquids, dense fluids and glasses, has been fascinating scientists for many years. The understanding of the liquid-to-glass transition mechanisms, thermal anomalies at low temperatures, divergence of transport properties and relaxation phenomena, is a challenge that is motivating strong experimental and theoretical efforts. In contrast to the crystalline case, in disordered systems the comprehension of atomic dynamics is complicated both due to the difficulties associated with the absence of translational invariance and due to the presence of other degrees of freedom, such as diffusion and relaxation processes in fluids. The presence of these processes automatically introduces different time-scales, $\tau$, which are usually strongly dependent on the specific thermodynamic state. These timescales affect the collective dynamical properties differently, depending on their value as compared to the time scale, $t_{\mathrm{D}}$, characterizing the vibrational dynamics of particles around their quasi-equilibrium positions. This is of the order of the inverse Debye frequency, i.e. comparable to that of a corresponding crystal with similar density and sound velocity. Moreover, one has to consider that the topological disorder introduces a second length scale, $\xi$, beside the interparticle distance, $\alpha$. As a matter of fact, the rich phenomenology observed in the dynamics of disordered systems can be ascribed to the interplay between these different structural $(\xi, \alpha)$ and dynamical $\left(\tau, t_{\mathrm{D}}\right)$ scales. An exhaustive understanding of dynamics in disordered systems in the so-called "mesoscopic region" (defined by length and time scales comparable to $\xi$ and $\tau$ ) is still not available and it represents a real challenge to modern physics [1]. A large amount of information can be experimentally deduced by determining the density-density correlation function, $F(Q, t)$, or, equivalently, of its time Fourier transform, the dynamic structure factor, $S(Q, E)$, in the largest momentum $(Q)$ and energy $(E)$ transfer region. Naturally, special attention should be paid to the portion of the $(Q, E)$-plane corresponding to the characteristic length-scales $(\xi, \alpha)$ and time-scales $\left(\tau, t_{\mathrm{D}}\right)$ of the system. In most materials $\xi$ and $\alpha$ are about a few tens and tenths of nanometers, respectively, while $t_{\mathrm{D}}$ is usually in the sub-picosecond range. On the other hand, $\tau$ can assume rather disparate values, since it strongly depends both on the specific nature of the relaxation process under consideration and on the thermodynamic conditions. However, the experience accumulated so far in this field points out 
that the relaxation processes that mainly influence the physical behavior of disordered systems are those with characteristic timescale of about $\xi / c_{\mathrm{S}}\left(c_{\mathrm{S}}\right.$ being the sound velocity). In most materials such a time scale falls in the $1 \div 10$ picosecond range. It is then natural to infer that, for studying the physics of disordered systems, the most important $(Q, E)$-range is the $0.05 \div 50 \mathrm{~nm}^{-1}$ and $0.01 \div 10 \mathrm{meV}$ ranges. In bulk materials $S(Q, E)$ can be directly measured by means of inelastic photon or thermal neutrons scattering experiments [2-5]. However, a single technique cannot completely cover, by itself, the entire range from inter-atomic distances to the continuum scale.

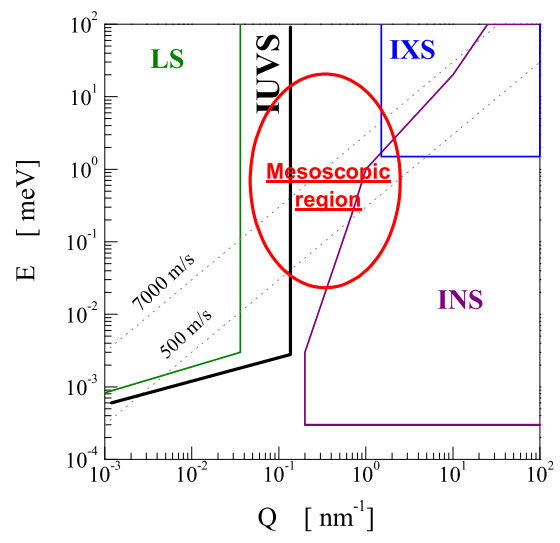

Figure 1. Kinematic regions accessible by existing techniques: Inelastic Light (LS), Ultra Violet (IUVS), X-ray (IXS) and thermal Neutron (INS) Scattering. The two dotted lines indicate energies of collective excitations with characteristic sound velocities of $500 \mathrm{~m} / \mathrm{s}$ and $7000 \mathrm{~m} / \mathrm{s}$.

Figure 1 displays the portion of the $(Q, E)$-plane where the major part of the condensed matter dynamics takes place, together with the regions where different experimental methods are currently available. The lines depict the characteristic energies of collective excitations propagating with two different sound velocities, typical of disordered systems. As can be evinced inspecting figure 1, the recently developed IUVS beamline at the ELETTRA synchrotron laboratory contributes to the reduction of the existing gap in the $(Q, E)$ regions accessed by LS and IXS/INS, thus enabling us to perform new investigations on the physics of liquid and glasses towards the mesoscopic region being of utmost interest. In the next section we will briefly describe the IUVS instrument. The following section reports the results of our recent investigations on liquid water, performed by IUVS as function of temperature and pressure at $Q$-values around $0.07 \mathrm{~nm}^{-1}$, a region of great interest, where peculiar dynamical behaviors are expected, as inferred by the results obtained through the complementary BLS and IXS techniques [6-10].

\section{The instrument}

In order to perform IUVS spectroscopy several requirements had to be fulfilled. Among them we cite the most demanding ones: incident photon flux on the sample larger than $10^{11}$ photons $/ \mathrm{s}$ in the photon energy region from 4 to $10 \mathrm{eV}$, and energy resolving power of about $10^{6}$. Due to the high photon flux, the radiation source has to keep the power harmful on the first optical elements. For such a reason we decided to use an exotic insertion device as radiation source, a Figure- 8 undulator [11], as an alternative to the standard vertical field devices. The main advantage of this solution is a much reduced on-axis power density, which is obtained with no penalty on the useful photon flux. Using a $32 \mathrm{~mm}$ period figure-8 undulator with maximum deflection parameters $K_{x}=3.4$ and $K_{y}=9.4$, at the exit of a $600 \times 600 \mathrm{mrad}^{2}$ pinhole the total power of the synchrotron radiation is as low as $20 \mathrm{~W}$ while the first harmonic delivers $2 \cdot 10^{15}$ photons $/ \mathrm{s} / 0.1 \% \mathrm{BW}$. The beam 
coming from the source has to be cleaned from the high order undulator harmonics and, for this reason, three reflections are used. More specifically the beam impinges on a gold coated GLIDCOP mirror internally water-cooled, which deviates the photons in the vertical plane with an angle of $6^{\circ}$. A second externally water-cooled silicon mirror is used to bring back the beam parallel to the floor. The beam is then focused by a spherical silicon mirror onto the entrance slits of the monochromator with a demagnification 20:1 and with an incident angle of $85^{\circ}$. Being the source size roughly $1 \times 1 \mathrm{~mm}^{2}$ (Horizontal $\times$ Vertical), a spot of $50 \times 20 \mu \mathrm{m}^{2}$ (vertically the astigmatism makes the focus larger) is obtained on the entrance of the monochromator. The only possible choice concerning the optical design of the monochromator, when a $10^{6}$ resolving power is needed at about $10 \mathrm{eV}$, is the Czerny-Turner model [12]. This design has the most desirable features, namely: better resolution, higher light-gathering power, simpler scanning mechanism and, furthermore, it presents the advantage of having fixed exit and entrance slits with no deviation in the direction of the exit beam. In this design light from the entrance slit is made parallel by a spherical concave mirror and is reflected onto an echelle plane grating. The grating used has 52 lines $/ \mathrm{mm}$ and works at a blaze angle of $69^{\circ}$. A second spherical mirror collects the diffracted beam and focuses it on the exit slit. The relative energy resolution, assuming that the intrinsic contribution coming from the grating is negligible, is given by the formula:

$$
\Delta E / E=\delta \cot \theta / 2 F
$$

where $\delta$ is the slit opening, $F$ is the focal length of the spherical mirror and $\theta$ is the blaze angle. We decided to built $8 \mathrm{~m}$ focal length monochromator to match the best compromise between the needed resolving power and mechanical feasibility, in fact, using $\delta=50 \mu \mathrm{m}, \mathrm{F}=8 \mathrm{~m}$ and $\theta=69^{\circ}$, we get a relative resolution of $\Delta E / E=1.1 \cdot 10^{-6}$. At the exit of the monochromator the beam impinges on a spherical mirror, which focuses the radiation onto the sample in a spot the size of about $30 \times 100 \mu \mathrm{m}^{2}$. A second spherical mirror is used to collect the radiation scattered from the sample and to send it into the entrance slit of the analyzer unit, having the same design as the monochromator. The inelastic scattering spectra are then collected by a low noise Peltiercooled CCD camera placed at the focal plane of the analyzer, which allows us to register the inelastic spectrum in one single shot, thus avoiding time-consuming monochromator scans of the diffraction angle. The quantum efficiency of the detector is larger than $10 \%$ for incident energies in the $5 \div 15 \mathrm{eV}$. The momentum transfer can be experimentally set either by changing the scattering angle, $\theta$, or the wavelength of the incident photons, $\lambda$, since it is given by the formula:

$$
Q=\frac{4 \pi n}{\lambda} \sin \left(\frac{\theta}{2}\right)
$$

where $\lambda$ and $n$ are the wavelength of the incident photons and the refraction index, respectively. The instrumental energy resolution has been measured by collecting the isotropic scattered intensity from a rough copper surface tilted with respect to the beam of about $40^{\circ}$. The measured instrumental energy resolution is $\Delta E / E=2 \cdot 10^{-6}$, very close to the theoretical expectation of $1.6 \cdot 10^{-6}$, given by the convolution of the energy resolutions of the analyzer and of the monochromator [13].

\section{The case of water}

Water has always occupied a unique role in the physics of liquids. Nevertheless its peculiar properties (such as the negative melting volume, the density maximum in the normal liquid range - which makes this substance so fundamental in life and earth - as well as the apparent divergence of the transport properties in the supercooled liquid region) depict an intriguing scenario still far from being well settled [14]. Different models have been proposed to explain this anomalous behavior: e.g., i) the existence of two liquid phases where a second critical point is located in the "no man's land" temperature region [15,16]; ii) the retracting spinoidal hypothesis, where thermodynamic anomalies are ascribed to the vicinity of the spinoidal line [17]; iii) a singularityfree scenario where thermodynamic anomalies can be ascribed to structural heterogeneities [18], and iv) the Mode Coupling Theory (MCT) which describes water features without resorting to 
an underlying thermodynamic singularity [19]. In this context, the need of experimental evidence, which can discriminate among these interpretative models, is evident. Although measurements of $S(Q, E)$ were performed by IXS [6,7] and BLS [8], a conclusive point was not reached. Basically, the reasons for that are twofold: a) the best sensitivity condition $\left(\omega_{\mathrm{p}} \tau \approx 1\right.$, where $\omega_{\mathrm{p}}$ is the frequency of the sound waves probed in the experiment and $\tau$ is the relaxation time) was never matched in BLS because of the low $\omega_{\mathrm{p}}$ values probed by this technique, while b) the lower $\omega_{\mathrm{p}}$ values probed by IXS are often too large in order to fulfil the $\omega_{\mathrm{p}} \tau \approx 1$ condition, especially in the most interesting case of supercooled water. On the other hand, in the latter case, IUVS frequency window matches the condition $\omega_{\mathrm{p}} \tau \approx 1$, allowing a precise determination of the relaxation parameters. Finally, in the case of IXS, the low throughput of the spectrometers does not allow us to reliably perform a detailed study of water as a function of thermodynamic parameters. Using IUVS we systematically measured $S(Q, E)$ of high purity water as a function of temperature (between 260 and $340 \mathrm{~K}$ ) and pressure (between 1 and $4000 \mathrm{bar}$ ). The latter measurements were performed at $5.1 \mathrm{eV}$ incident photon energy $\left(Q \approx 0.07 \mathrm{~nm}^{-1}\right)$, while room pressure measurements were performed both at $5.1 \mathrm{eV}$ and $6.7 \mathrm{eV}$, corresponding to Q-values of about $0.09 \mathrm{~nm}^{-1}$ [20]. In what follows we will discuss two examples of the results one can obtain by using IUVS in the study of water. In particular, the temperature (pressure) dependence of the structural relaxation time in water at fixed pressure (temperature) is discussed.

\subsection{Room pressure water vs. temperature}

In figure 1 we show a selection of IUVS spectra of liquid and supercooled water at room pressure. The clear broadening of the Brillouin peak - which is not resolution limited, as emphasized by superimposing the resolution function on the spectrum at $287.1 \mathrm{~K}$ - strongly increases with decreasing temperature, suggesting the presence of a relaxation process whose time scale matches the experimental frequency window. We analyzed the measured spectra, $I(Q, E)$, by fitting the experimental data with a model function properly convoluted with the intrinsic energy resolution function of the spectrometer, $R(E)$, scaled by an overall intensity factor, $A$. Finally, a flat background contribution, $B$, was added:

$$
I(Q, E)=A \cdot S(Q, E) \otimes R(E)+B
$$

where $\otimes$ is the convolution integral. The model function used in describing $S(Q, E)$ was derived within the memory function formalism, in this framework $S(Q, E)$ can be written as [2]:

$$
S(Q, E)=\frac{\left(\hbar c_{\mathrm{s}} Q\right)^{2} m^{\prime}(Q, E)}{\left[\left(E^{2}-\left(\hbar c_{\mathrm{s}} Q\right)^{2}-E m^{\prime \prime}(Q, E)\right]^{2}+\left[E m^{\prime}(Q, E)\right]^{2}\right.},
$$

where $c_{\mathrm{s}}$ is the adiabatic sound velocity while $m^{\prime}(Q, E)$ and $m^{\prime \prime}(Q, E)$ are the real and imaginary part of the time Fourier transform of the so-called memory function: $m(Q, t)$. In deriving equation (3) the assumption that the specific heat ratio, $\gamma$, is equal to 1 has been made. In this case the effect of thermal diffusion process on the shape of $S(Q, E)$ can be neglected [21]. Such an approximation is particularly suited for water since in this case $\gamma$ is only a few percent different from 1 ; further details regarding this kind of data analysis are reported elsewhere $[6,7]$. The analytical form we used for the memory function was chosen to be consistent with the viscoelastic framework, which has been already proved in order to satisfactorily describe the collective dynamics of water [6-10,22] and other fluid systems: from supercooled glass formers [23] up to compressed gases $[24,25]$. Within this framework the time dependence of the memory function has, at least, one time decay (i.e. a relaxation) with a finite time scale. The simpler choice is thus as follows:

$$
m(Q, E)=\Delta \exp (-t / \tau),
$$

where $\Delta$ and $\tau$ are usually referred to as relaxation strength and time, respectively. The relaxation function reported in equation (4) is quite a crude approximation being the relaxation process characterized by a single time scale. A continuous distribution of relaxation times is, indeed, a 


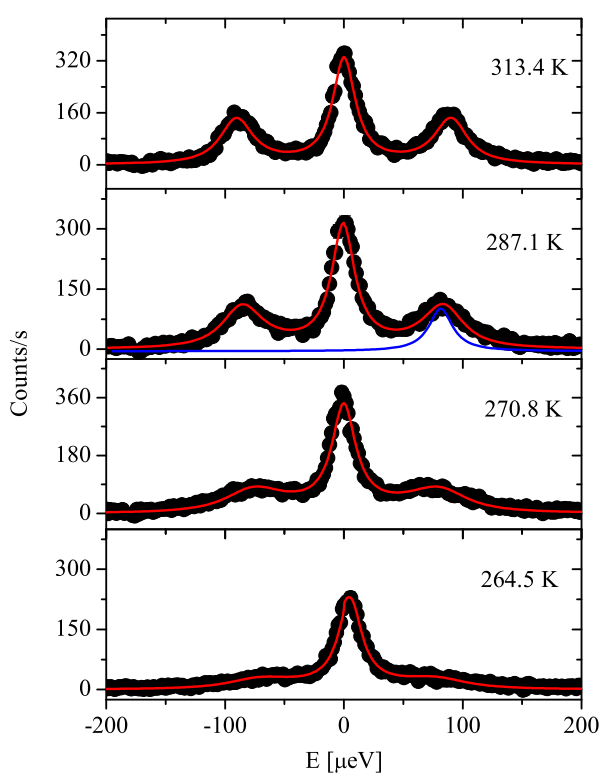

Figure 2. Selection of IUVS spectra of liquid and supercooled water (dots), taken at $6.7 \mathrm{eV}$ incident photon energy $\left(Q \approx 0.09 \mathrm{~nm}^{-1}\right)$, at the indicated temperatures. The fit results are superimposed (red lines). The blue line in the spectra at $287.1 \mathrm{~K}$ is the experimental energy resolution function.

more reliable approximation. In particular, according to $\mathrm{MCT}$, the relaxation behavior of $m(Q, t)$ can be approximated by the following equation:

$$
m(Q, E)=\Delta \exp \left(-\left(t / \tau_{\mathrm{MCT}}\right)^{\beta}\right),
$$

that represents a distribution of relaxation functions as the one reported in equation (4). $\tau_{\mathrm{MCT}}$ is the $\tau$-value corresponding to the case when the distribution assumes its maximum while $\beta$, commonly referred to as stretching parameter, is related to the broadening of such a distribution. The average relaxation time, $\langle\tau\rangle$, associated to the relaxation function reported in equation (5) is given by $\langle\tau\rangle=\beta \tau_{\mathrm{MCT}}$, while in case of equation (4) it is simply equal to $\tau$. Hereafter we will refer to $\langle\tau\rangle$ simply as relaxation time and we will indicate it as $\tau$.

MCT foresees that: (i) $\beta<1$, experimentally found to be $\approx 0.6$, and (ii) $\tau$ follows a power law divergence as a function of temperature [18]:

$$
\tau(T)=\left(T-T_{\mathrm{MCT}}\right)^{-\delta} .
$$

MCT calculations and Molecular Dynamics simulations [22] have estimated the divergence temperature $T_{\mathrm{MCT}}$ of water to be of about $220 \mathrm{~K}$ and $\delta=2.3 \pm 0.2$.

By fitting IUVS spectra with the model function obtained inserting the Fourier transform of equation (5) into equation (3), we determined the temperature dependence of both the structural relaxation time and the stretching parameter of liquid and supercooled water. The obtained values are displayed in figure 3. Concerning the relaxation time, the comparison to IXS results of [6] shows a good agreement between the two techniques above the room temperature, despite the two different models used for the memory function. In fact IXS data were fitted using the simple exponential time decay for the relaxation (equation (4)) instead of the stretched one (equation (5)). The temperature dependence of $\tau$, either determined by IXS or IUVS, can be empirically described by an activation (Arrhenius) law:

$$
\tau(T)=\exp \left(E_{\mathrm{a}} / k_{\mathrm{B}} T\right)
$$


where $E_{\mathrm{a}}$ and $k_{\mathrm{B}}$ are the activation energy of the relaxation and the Boltzmann constant, respectively. One can derive the $E_{\text {a }}$ value $(23 \pm 2 \mathrm{~kJ} / \mathrm{mol})$ by fitting IUVS data with equation (7) which is about the activation energy of water hydrogen bonds: i.e. $23 \mathrm{~kJ} / \mathrm{mol}$ [26]. Despite the quite different temperature range explored in previous IXS experiments [6], discrete agreement with these results $\left(E_{\mathrm{a}}=16 \pm 3 \mathrm{~kJ} / \mathrm{mol}\right)$ is found as well. It is then possible to conclude that the relaxation process is related to the making and breaking of hydrogen bond network in water.

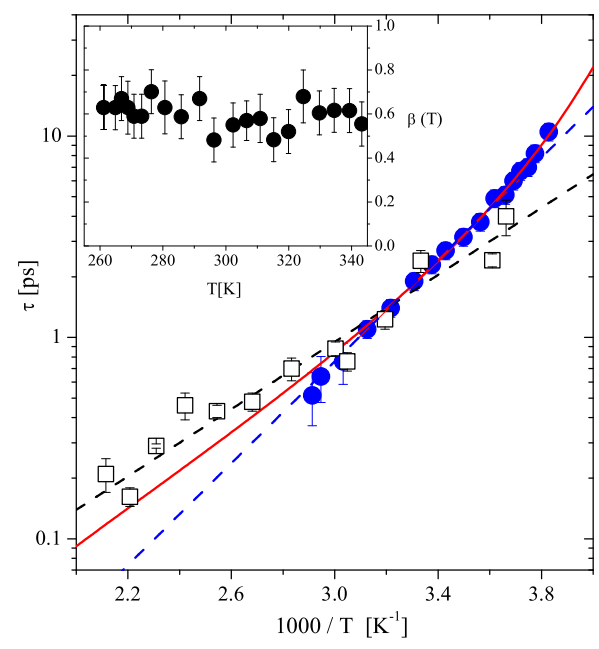

Figure 3. Structural relaxation time of water as a function of inverse temperature. Here we compare our IUVS results (solid blue circles) with IXS measurement (open squares [6]). Both IXS and IUVS data, separately, can be interpreted as an Arrhenius behavior (dotted lines). The whole data sets (IXS + IUVS) can be also described by a power-law divergence of $\tau$ towards $220 \mathrm{~K}$ (solid line), in good agreement with MCT predictions. In the inset we also highlight the temperature independence of $\beta$, as foreseen by MCT.

Inspecting figure 3 one can also appreciate that the whole data set of IXS and IUVS data can be also described by the power law predicted by MCT (equation (6)). The power law diverges at $T_{\mathrm{MCT}}=220 \pm 10 \mathrm{~K}$, and has an exponent $\delta=2.3 \pm 0.2$, in good agreement with previous determinations [22]. Moreover, the stretching parameter is temperature independent and close to 0.6 , consistently with MCT predictions [19]. Therefore these results support the interpretation of a purely dynamic origin for the divergence of $\tau$, releasing the need of an underlying thermodynamic singularity for its explanation. However, it has to be pointed out that IXS and IUVS experiments were not performed in the same thermodynamics conditions. In particular IXS experiment was performed at a constant density $\left(1 \mathrm{~g} / \mathrm{cm}^{3}\right)$, while the IUVS one was performed at constant pressure (1 bar). Consequently, eventual explicit pressure/density dependencies of $\tau$ could play a role in explaining the experimental data reported in figure 3. With the aim of clarifying this point we decided to employ IUVS in order to systematically investigate the pressure/density behavior of $\tau$; highlights of the preliminary results are reported further below.

\subsection{Water at $273 \mathrm{~K}$ vs. pressure}

In order to study the relaxation phenomenology as a function of pressure, a high-pressure device based on a hand pump pressure generator and a hydrostatic pressure cell have been employed. Details about this setup can be found elsewhere [25]. We used this kind of sample environment in order to collect IUVS spectra of water at $273 \mathrm{~K}$ as a function of pressure in the 1-4000 bar range. In figure 4 a selection of IUVS spectra of liquid water at $273 \mathrm{~K}$ at different pressures are reported. Analogously to the ones shown in figure 4, a broadening of Brillouin peaks larger than the resolution 
function was always noticed. Moreover, differently from room pressure data, an intense quasi-elastic spectral component was observed in the spectra $(\approx 90 \%$ of the whole spectral intensity). This strong central peak arises from spurious scattering by the optical windows of the pressure cell and does not permit to perform a reliable fitting procedure, as the one we did in the case of room pressure water. However, manipulating equations (3) and (4), after some straightforward algebra, it is possible to relate the energy position, $E_{\mathrm{MAX}}$, of the maxima of longitudinal current spectra, $J_{\mathrm{L}}(Q, E)=(E / Q \hbar)^{2} S(Q, E)$, to the relaxation parameters, $\tau$ and $\Delta$, or, alternatively, it is possible to express $\tau$ as a function of $E_{\mathrm{MAX}}$ and $\Delta$ :

$$
\tau=\sqrt{\frac{1-\left(c_{\mathrm{s}} \hbar Q / E_{\mathrm{MAX}}\right)^{4}}{2 \Delta^{2}-2\left(\hbar c_{\mathrm{s}} Q\right)^{2}-2 E_{\mathrm{MAX}}^{2}}} .
$$

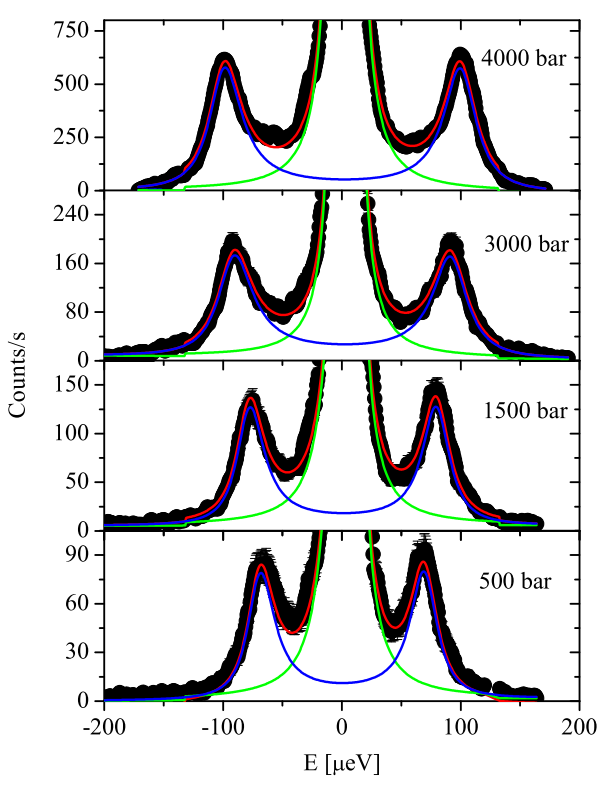

Figure 4. Selection of IUVS spectra of liquid water at $273 \mathrm{~K}$ at the indicated pressures (dots). Data were taken at $5.1 \mathrm{eV}$ incident photon energy $\left(Q \approx 0.07 \mathrm{~nm}^{-1}\right)$. The fit results are superimposed (blue lines: DHO function; green lines: Lorentian function accounting for the spurious central peak; red lines: sum).

The values of $E_{\text {MAx }}$ can be determined by fitting IUVS spectra with an empirical model consisting in a DHO function describing the Brillouin peaks plus a Lorentian function centered at $E=0$ accounting for the central spurious component:

$$
S(Q, E) \propto \frac{A_{\mathrm{L}}}{E^{2}+W^{2}}+A_{\mathrm{DHO}} \frac{E_{\mathrm{MAX}}^{2} \Gamma}{\left(E^{2}-E_{\mathrm{MAX}}^{2}\right)^{2}+(E \Gamma)^{2}},
$$

where $\Gamma$ and $W$ are the widths of Brillouin and central peaks, respectively, while $A_{\mathrm{L}}$ and $A_{\mathrm{DHO}}$ are intensity factors. On the other hand, in the pressure and temperature ranges where IUVS experiments were performed, the parameter $\Delta$ can be quite accurately estimated by the numerous IXS experiments performed so far $[6,7,9,27]$. This alternative strategy for the determination of $\tau$ can be also applied for room pressure data. As one can appreciate from the inset of figure 5 , the values of $\tau$ determined with the two different procedures are fully consistent with each other, thus providing an excellent consistency check of the employed procedure. It is worth stressing that the data shown in the inset of figure 5 (open squares) correspond both to old spectra taken at $6.7 \mathrm{eV}$ 
incident photon energy, as the ones shown in figure 2 but analyzed using equations (8) and (9), as well as to new spectra recently taken at $5.1 \mathrm{eV}$ incident photon energy.

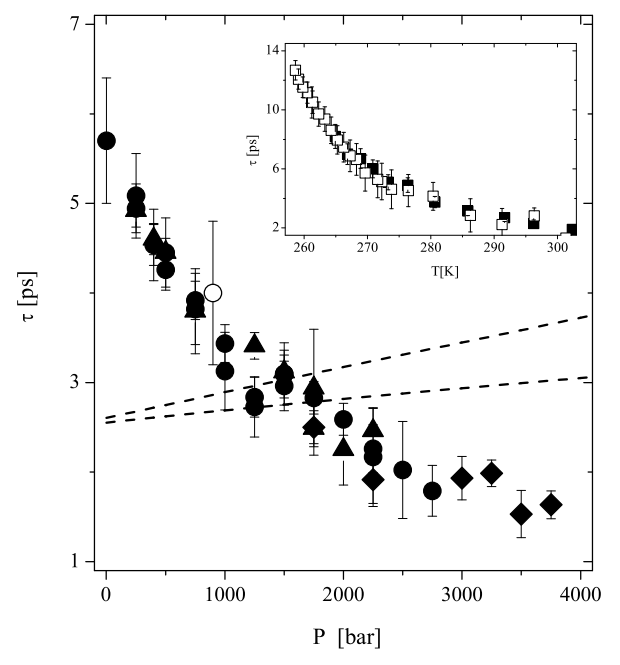

Figure 5. Structural relaxation time of water as a function of pressure (solid symbols). The open circle is the only available IXS datum (from [6]). The full lines depict the expected trend, according to equation (10) (the stepper line corresponds to $\gamma=1$, while the other corresponds to $\gamma=0.5$, see text for further details). In the inset we show the data of room pressure water as a function of temperature as obtained either by direct fitting IUVS spectra with equations (3) and (5) (full squares) or by using equations 8 and 9 (open squares).

The pressure dependence of relaxation time is reported in the main panel of figure 5; different symbols stand for different data sets, corresponding to different experiments roughly performed 3 months one after the other. We can immediately observe that IUVS data sets are in excellent agreement between them and with the only available IXS datum. Even from a first inspection of this figure, one can appreciate that $\tau$ decreases with increasing pressure. This trend is quite surprising since density increases of about $14 \%$ from 1 to 4000 bar and, simply considering the reduction of the free volume, a non-negligible increase of $\tau$ is expected with increasing pressure. In fact, using general arguments it is possible to derive the following equation for the temperature and volume (i.e. density) dependence of $\tau$ [28]:

$$
\tau=\exp \left(E_{\mathrm{a}} / k_{\mathrm{B}} T+\alpha v_{0} /\left(v-v_{0}\right)\right),
$$

where $v$ is the specific volume, $v_{0}$ is the volume of water molecules and $\alpha$ is a positive numerical factor (usually between 0.5 and 1 ) accounting for the possible overlapping of volumes associated to different molecules. A comment is deserved: in principle, also in the case of room pressure data we need to account for the reduction of the free volume $\left(v_{\mathrm{f}}=v-v_{0}\right)$ but, since the density changes only of about $1 \%$ in the probed temperature range, it can be safely neglected.

The excellent consistency between these methods highlights the soundness of the procedure used for deriving the values of $\tau$ in the case of water under high pressure.

In conclusion, according to equation (10), the data shown in figure 5 suggest that $E_{\text {a }}$ presents a remarkable density dependence or, alternatively, that a temperature/volume behavior as the one reported in equation (10) cannot describe those experimental results. Nevertheless, further investigations are needed in order to better clarify these preliminary experimental data and to cast them in an assessed framework. We are currently performing systematic IUVS measurements in the pressure and temperature ranges of 1-5000 bar and 250-350 K with the aim to fully characterize the density and temperature dependencies of $\tau$. 


\section{Conclusions}

We have shown the capability of inelastic UV scattering to study collective dynamics of disordered systems in a kinematic region not accessible before. In the case of water we determined the temperature dependencies of the structural relaxation time and stretching parameter in the region where the transport properties start to diverge (i.e. in the supercooled liquid phase). Our experimental results agree with mode coupling theory predictions suggesting that, at ambient pressure, the divergence in transport properties is of a dynamic origin. We have also shown the capabilities of IUVS to be coupled with high-pressure hydrostatic devices and, therefore, to determine the values of relaxation time as a function of pressure. We noticed that the density dependence $\tau$ cannot be accounted for by a simple reduction of the free volume. One possible explanation of the observed behavior is that the activation energy of the relaxation is volume dependent or, alternatively, that other physical processes could effect the observed relaxation behavior.

\section{References}

1. Jackle J., Reports on Progress in Physics, 1986, 49, 171. For recent reviews, see, for instance, Proceedings to the 3rd Workshop on Nonequilibrium Phenomena in Supercooled Fluids, Glasses and Amorphous Materials, Pisa Italy, 2002 Journal of Physics of Condensed Matter; 15, (2003); Proceedings to the 4th International Discussion Meeting in Relaxations in Complex Systems, Hersonissos, Crete, 2001 Journal of Non-Crystalline Solid 307-310, 1-1080 (2002); Proceedings to the 8th International Workshop on Disordered Systems, Andalo, Italy, 2001, Philosophical Magazine 82, No. 3 (2002).

2. Boon J.P., Yip S. Molecular Hydrodynamics. McGraw-Hill, New York, 1980.

3. Berne B.J., Pecora R. Dynamic Light Scattering with applications to chemistry, biology and physics. Wiley, New York, 1976.

4. Marshall W., Lovesey S.W. Theory of Thermal Neutron Scattering. Clarendon Press, Oxford, 1971.

5. Burkel E., Report on Progress in Physics, 2000, 63, 171.

6. Monaco G., Cunsolo A., Ruocco G., Sette F., Physical Review E, 1999, 60, 5505.

7. Bencivenga F., Cunsolo A., Krisch M., Monaco G., Ruocco G., Sette F., Physical Review E, 2007, 75, 051202 .

8. Cunsolo A., Nardone M., Journal of Chemical Physics, 1998, 105, 3911.

9. Ruocco G., Sette F., Leonardo R.Di., Fioretto D., Krisch M., Lorentzen M., Masciovecchio C., Monaco G., Pignon F., Scopigno T., Physical Review Letters, 1999, 83, 5583.

10. Vacher R., Pelous J., Physical Review B, 1976, 14, 823.

11. Tanaka T., Kitamura H., Nucl. Instr. and Meth. in Phys. Res. A, 1995, 364, 368.

12. Czerny M., Turner A.F., Z. Physik, 1930, 61, 792.

13. Masciovecchio C., Cocco D., Gessini A. Proceedings of $8^{\text {th }}$ International Conference of Synchrotron Radiation Instrumentation. San Francisco, California, 25-29 Aug 2003, Warwick, American Institute of Physics, 2004, 1190.

14. Mishima O., Stanley E. Nature, 1998, 396, 329.

15. Poole P.H., Sciortino F., Essmann U., Stanley H.E., Nature, 1992, 360, 324.

16. Mishima O., Stanley H.E., Nature, 1998, 392, 164.

17. Speedy R.J., Journal of Chemical Physics, 1982, 86, 982.

18. Poole P.H., Sciortino F., Grande T., Stanley H.E., Angell C.A., Physical Review Letters, 1994, 73, 1632; Truskett T.M., Debenedetti P.G., Sastry S., Torquato S., Journal of Chemical Physics, 1999, 111, 2647.

19. Goetze W., Sjogren L., Reports on Progress in Physics, 1992, 55, 241.

20. Masciovecchio C., Santucci S.C., Gessini A., Di Fonzo S., Ruocco G., Sette F., Physical Review Letters, 2004, 92, 255507.

21. Bencivenga F., Cunsolo A., Krisch M., Monaco G., Ruocco G., Sette F., Europhysics Letters, 2006, $\mathbf{7 5}, 70$.

22. Sciortino F., Gallo P., Tartaglia P., Chen S.H., Physical Review Letters, 1996, 76, 2733. Sciortino F., Gallo P., Tartaglia P., Chen S.H., Physical Review E, 1996, 54, 6331. Starr F.W., Bellissent-Funel M.C., Stanley H.E., Physical Review Letters, 1999, 82, 3629. Starr F.W., Sciortino F., Stanley H.E., Physical Review E, 1999, 60, 6757.

23. Fioretto D., Comez L., Socino G., Verdini L., Corezzi S., Rolla P.A., Physical Review E, 1999, 59, 1899. 
24. Cunsolo A., Pratesi G., Verbeni R., Colonnesi D., Masciovecchio C., Monaco G., Ruocco G., Sette F., Journal of Chemical Physics, 2001, 114, 2259.

25. Bencivenga F., Cunsolo A., Krisch M., Monaco G., Orsingher L., Ruocco G., Sette F., Vispa A., Physical Review Letters 98, 085501, (2007).

26. Angell C.A. Annual Review of Physical Chemistry, 34, 593, (1983); in Water: a Comprehensive Treatise, edited by F. Franks, Plenum Press, New York, (1972), Vol. 7.

27. Krisch M., Loubeyre P., Ruocco G., Sette F., Cunsolo A., Dtastuto M., LeToullec R., Lorenzen M., Mermet A., Monaco G., Verbeni R., Physical Review Letters, 89, 125502, (2002).

28. Macedo P.B., Litovitz T.A., The Journal of Chemical Physics 42, 2451, (1965).

\title{
Динаміка води на наномасштабах
}

\author{
К.Машіовеккіо, Ф.Бенчівенґа, А.Ґессіні \\ Синхротрон Трієсту, Трієст, Італія
}

Отримано 12 листопада 2007 р.

\begin{abstract}
Недавня побудова лінії непружнього розсіяння ультрафіолетових променів (НРУп) у синхротронній лабораторії ELETTRA відкриває нові можливості для дослідження спектру флуктуацій густини, $S(Q, E)$, у невпорядкованих системах у мезоскопічній області передачі імпульсу $(Q)$ та енергії $(E)$, яка $є$ недоступною для інших спектроскопічних методик. Як приклад можливого застосування методу НРУП ми обговорюємо нові результати для випадку динаміки води. 3 аналізу спектрів НРУП ми мали можливість виміряти залежність від температури та тиску для часу структурної релаксації $(\tau)$ у воді. Для випадку нормального тиску значення $\tau$, визначене з НРУП, добре узгоджується 3 попередніми розрахунками та, що є більш важливо, його температурна залежність узгоджується 3 передбаченнями теорії взаємодіючих мод. Крім того, ми знайшли, що $\tau$ зменшується зі зростанням тиску при фіксованій температурі. Спостережувана тенденція демонструє, що явище структурної релаксації має сильну залежність від прикладеного тиску. Однак, для того, щоб вияснити фізичне значення цих попередніх експериментальних результатів, необхідні подальші дослідження.
\end{abstract}

Ключові слова: вода, непружнє розсіяння, релаксація

PACS: $78.35 .+c, 62.60 .+v, 62.50 .+p$ 